\title{
武汉后湖长角涵螺的周年生产量”
}

\author{
间云君 ${ }^{12}$ 梁彦龄 ${ }^{2}$
}

(1 华中科技大学生命科学与技术学院, 武汉 430074,2 :中国科学院水生生物研究所, 武汉 430072)

提 要 采用两种方法对后湖长角涵螺 (Alocinma longicornis) 的周年生产量进行了 测算 结果表明, 两种方法得到的生产量较为吻合.生产量的带壳湿重和去壳干重分别是 : 体长频率法 $2.22 \mathrm{~g} /\left(\mathrm{m}^{2}\right.$ a) $0.111 \mathrm{~g} /\left(\mathrm{m}^{2}\right.$ a) ; 瞬时生长率法 $2.177 \mathrm{~g} /\left(\mathrm{m}^{2}\right.$ a) $) 0.109 \mathrm{~g} /\left(\mathrm{m}^{2}\right.$ a). 对应的 $\mathrm{P} / \mathrm{B}$ 系数为 4.05 .4 .

关键词 后湖 长角涵螺 生产量 $\mathrm{P} / \mathrm{B}$ 系数

分类号 Q959.121 ${ }^{+} .1$

长角涵螺是我国浅水湖泊常见的一种优势螺类 ${ }^{[1 \sim 4]}$, 密度大, 生物量可观, 不仅是青鱼、鲤鱼等经济鱼类 的优质天然饵料, 而且在湖泊物质循环和能量流动中起着重要作用, 其功能的重要标志一一生产力国内外至 今尚无报道. 为了了解长角涵螺在我国湖泊中的生态功能和渔产潜力, 作者于 1996 年 4 月至 1997 年 3 月对武 汉后湖的长角涵螺进行了周年逐月采集,并采用体长频率法和瞬时生长率法测算了周年生产量.

\section{1 工作方法}

\section{1 采集点和采样时间}

由于后湖底泥较为均一, 底栖动物分布较随机, 因此在湖心截面上共设 4 个间距相等的采样点(图 1).采 样时间从 1996 年 4 月至 1997 年 3 月，一般在每月 15 日左右。

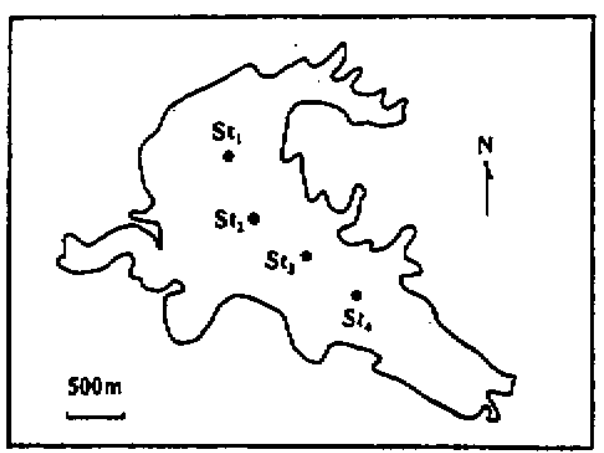

图 1 后湖及采样点的分布

Fig. 1 Lake Houhu and distribution of sampling sites

\section{2 标本采集及处理}

定量采集使用 $1 / 16 \mathrm{~m}^{2}$ 改良彼得生式采泥器, 每点一 次, 个别两次, 泥样经 60 目铜篮或纱网篮洗后, 置于白色 解剖盘中分检 标本用 $10 \%$ 的福尔马林固定.

\section{3 生产量的测算}

计数、测量每月个体数和壳长, 结合已获得的体长 - 体重关系方程计算干、湿重.采用体长频率法和瞬时 生长率法测算了周年生产量.

\section{2 结果}

\section{1 种群动态}

长角涵螺的种群动态包括数量动态和生物量动态.

2.1 .1 数量动态 后湖长角涵螺种群密度 (图 2) 在其繁 殖高峰期四月最大达到 $68 \mathrm{ind} / \mathrm{m}^{2}$. 此后快速下降, 9、10 月最低降至 $4 \mathrm{ind} / \mathrm{m}^{2} .12$ 月再次出现次高峰, 这可能是 由于长角涵螺趋向于在湖心冬眠，容易采集所致.次年 3 月定量样本中没有采到长角涵螺标本.

2.1 .2 生物量动态 后湖长角涵螺生物量在十二月份出现峰值, 为 $4.80 \mathrm{~g} / \mathrm{m}^{2}$. 由于三月份在定量样本中没有 采到标本，生物量被认为是 0 (图 3). 


\section{2 生长}

从图 4 可以看出, 1996 年 4、5 月产出的长角涵 螺在 11 月份达到成体体长, 而 1995 年出生的少数 个体可以存活到 1996 年 10 月以后. 因此可以推出 后湖长角涵螺的生活史为一年到一年半.

\section{3 长角涵螺的周年生产量}

后湖长角涵螺的生产量的测算采用瞬时生长 率法(Instantaneous growth rate method)和体长频率法 ( Size-frequency method)

2.3.1 瞬时生长率法 瞬时生长率法测算长角涵 螺的周年生产量(带壳湿重). 从表 1 可知, 后湖长 角涵螺的周年生产量为 $2.177 \mathrm{~g} / \mathrm{m}^{2}, \mathrm{P} / \mathrm{B}$ 系数为 5 . 4. 其软体部分湿重占个体湿重的 $33.4 \%$,软体部 分的干湿比为 0.15 ,因此后湖长角涵螺的周年生 产量 (去壳干重)为 $2.177 \times 33.4 \% \times 0.15=0.109$ $\mathrm{g} / \mathrm{m}^{2}, \mathrm{P} / \mathrm{B}$ 系数为 5.4 .

2.3.2 体长频率法 (Size-frequency method) 体长频 率法测算长角涵螺的周年生产量 (表 2), 可知, 体 长频率法测算的后湖长角涵螺周年生产量 (带壳 湿重) 为 $2.22 \mathrm{~g} / \mathrm{m}^{2}, \mathrm{P} / \mathrm{B}$ 系数为 4.0 . 其周年生产量 (去壳干重) 为 $0.111 \mathrm{~g} / \mathrm{m}^{2}, \mathrm{P} / \mathrm{B}$ 系数为 4.0 .

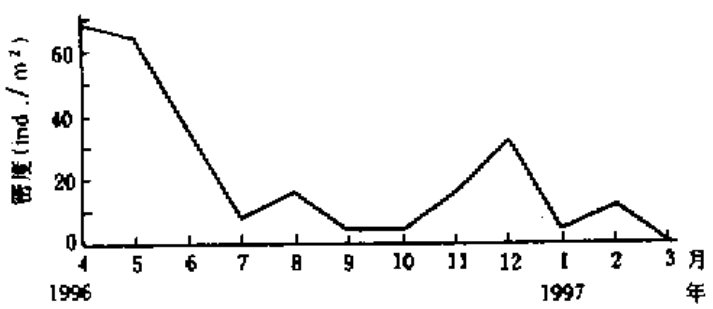

图 2 后湖长角涵螺种群的密度动态

Fig. 2 Dynamics of density of $A$. longicornis population in Lake Houhu

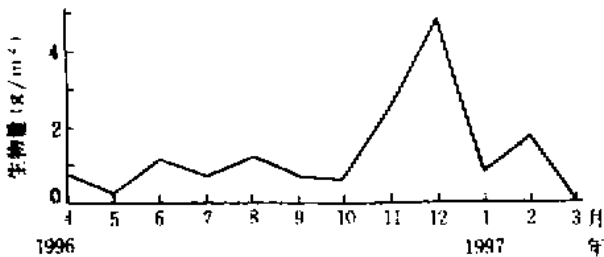

图 3 后湖长角涵螺的生物量动态

Fig. 3 Dynamics of biomass of $A$. longicornis population in Lake Houhu

表 1 瞬时生长率法测算后湖长角涵螺的周年生产量 (带壳湿重)

Tab. 1 Annual production ( $\mathrm{mg} / \mathrm{m}^{2}$ in wet wt with shell) of $A$. longicornis in Lake Houhu calculated by instantaneous growth rate method

\begin{tabular}{ccccccc}
\hline 月份 & 4 & 5 & 6 & 7 & 8 & 11 \\
\hline 个体均重 $(\mathrm{mg})$ & 1.316 & 4.080 & 16.93 & 15.54 & 45.14 & 154.40 \\
平均现存量 $\left(\mathrm{mg} / \mathrm{m}^{2}\right)$ & 172.68 & 401.44 & 301.96 & 301.92 & 826.68 & 413.34 \\
瞬时生长率 & 1.132 & 1.423 & -0.086 & 1.066 & 1.230 & 0.237 \\
生产量 $\left(\mathrm{mg} / \mathrm{m}^{2}\right)$ & 195.47 & 571.24 & -25.96 & 321.84 & 1016.80 & 97.96 \\
\hline
\end{tabular}

由此可见，两种方法测算的周年生产量较为吻合, $\mathrm{P} / \mathrm{B}$ 系数有一定差异，主要原因可能是两种方法在计算 平均现存量时存在一些差异而致.

表 2 瞬时生长率法测算后湖长角涵螺的周年生产量 (带壳湿重)

Tab. 2 Annual production $\left(\mathrm{mg} / \mathrm{m}^{2}\right.$ in wet wt with shell) of $A$. longicornis in Lake Houhu calculated by size-frequency method

\begin{tabular}{|c|c|c|c|c|c|c|c|}
\hline 体长组 & 密度 & 体均重 & 生物量 & 减员数 & 减员个体均重 & 损失量 & 生产量 \\
\hline$(\mathrm{mm})$ & (ind $/ \mathrm{m}^{2}$ ) & $(\mathrm{mg})$ & $\left(\mathrm{g} / \mathrm{m}^{2}\right)$ & (ind $/ \mathrm{m}^{2}$ ) & $(\mathrm{mg})$ & $\left(\mathrm{g} / \mathrm{m}^{2}\right)$ & $\left(\mathrm{g} / \mathrm{m}^{2}\right)$ \\
\hline $1 \sim 2$ & 5.3 & 0.781 & 0.0041 & 1.0 & 1.797 & 0.0018 & \\
\hline $2 \sim 3$ & 4.3 & 4.137 & 0.0179 & 1.3 & 7.161 & 0.0096 & 0.0768 \\
\hline $3 \sim 4$ & 3.0 & 12.404 & 0.0372 & 2.7 & 18.691 & 0.0312 & 0.2496 \\
\hline $4 \sim 5$ & 1.3 & 28.164 & 0.0376 & 0.6 & 39.074 & 0.0260 & 0.2080 \\
\hline $5 \sim 6$ & 0.7 & 54.210 & 0.0361 & 0.0 & 71.194 & 0.000 & 0.0000 \\
\hline $6 \sim 7$ & 0.7 & 93.500 & 0.0623 & -0.8 & 118.090 & -0.0945 & -0.7560 \\
\hline $7 \sim 8$ & 1.5 & 149.146 & 0.2237 & 0.8 & 182.936 & 0.1463 & 1.1704 \\
\hline $8 \sim 9$ & 0.7 & 224.381 & 0.1571 & 0.7 & 224.381 & 0.1571 & 1. 2568 \\
\hline
\end{tabular}




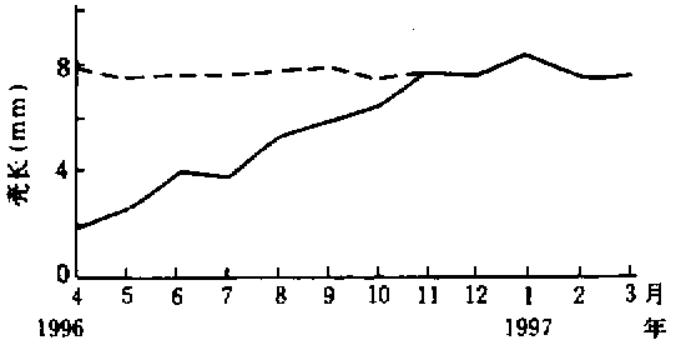

图 4 后湖长角涵螺的生长曲线

Fig.4 Growth curve of A. Longicornis in Lake Houhu

\section{3 讨论}

长角涵螺的生产量国内外尚无报道.其生活 史与纹沼螺相似 ${ }^{[6]}$,为一年到一年半 , $\mathrm{P} / \mathrm{B}$ 系数平 均为 4.7 , 与 Water ${ }^{[7]}$ 列举的 Melania tuberculata， Bellamya nuicolor、Planorbis vortex 等螺类相比生活 史近似, 生产量(去壳干重) 差异也不大 ,P/B 系数 比较一致.说明这些螺类的生产量受生活史影响 较生境更大, 可能是由于生活史决定了这类动物 的生长时间长短，从而间接决定成年个体的大小， 而生境主要影响生长快慢。

\title{
参 考 文 献
}

1 梁彦龄, 吴天惠, 谢志才. 保安湖底栖动物现状及渔业评价. 梁彦龄等主编 草型湖泊资源. 环境与渔业生态学管理 $($ 一). 北京 科学出版社, $1995: 178 \sim 192$

2 梁彦龄, 吴天惠, 谢志才. 西凉湖底栖动物及渔产潜力估算的初步研究. 梁彦龄等主编 草型湖泊资源、环境与渔业生态学管理 (一). 北京 : 科学出版社 , 1995: 194 203

3 陈其羽, 谢翠娴 梁彦龄等. 望天湖底栖动物种群密度与季节变动的初步观察.海洋与湖沼 ,1982,13(1) 78 86

4 陈其羽.湖北省花马湖软体动物的调查报告.海洋与湖沼, 1979, 10(1) : 46 ～62

5 Hunter R D. Growth , fecundity , and bioenergetics in three populations of Lymnaea palustris in upstate New York. Ecology , $1975,5656 \sim 63$

6 问云君 梁彦龄 ,王洪铸.保安湖螺类生产力的研究, II. 纹沼螺的周年生产量.水生生物学报, 2001 25(1) 36 41

7 Water T F. Secondary Production in Inland Waters. Adv Ecol Res , 1977,10 : 91 - 164

\section{Annual Production of Alocinma longicornis in Lake Houhu (Wuhan , China)}

\author{
YAN Yunjun $^{12} \quad$ LIANG Yanling ${ }^{2}$ \\ ( 1 :School of life and Technology , Huazhong University of Science and Technology , Wuhan 430074 , P. R. China ; \\ 2 :Institute of Hydrobiology, Chinese Academy of Sciences Wuhan 430072 , P. R. China)
}

\begin{abstract}
The production of Alocinma Longicornis in Lake Houhu was estimated by two method. The results are $: 2.22 \mathrm{~g} /\left(\mathrm{m}^{2}\right.$ a) of wet weight with shell or $0.111 \mathrm{~g} /\left(\mathrm{m}^{2}\right.$ a) of dry weight shell-free by size-frequency method $; 2.177 \mathrm{~g} / \mathrm{m}^{2}$ a of wet weight with shell or $0.109 \mathrm{~g} / \mathrm{m}^{2}$ a of dry weight shell-free by instantaneous growth method. The corresponding $\mathrm{P} / \mathrm{B}$ ratio are 4.0 , 5.4 ,respectively.
\end{abstract}

Keywords :Lake Houhu ; A. Longicornis ; production ; P/B ratio 